\title{
(2) OPEN ACCESS \\ Unequal impact of the COVID-19 crisis on minority ethnic groups: a framework for understanding and addressing inequalities
}

\author{
Srinivasa Vittal Katikireddi (D) ,' Sham Lal (D) ,' ${ }^{2}$ Enitan D Carrol, ${ }^{3}$ \\ Claire L Niedzwiedz (D) , ${ }^{4}$ Kamlesh Khunti, ${ }^{5}$ Ruth Dundas (D) , ${ }^{1}$ Finn Diderichsen (D) , \\ Ben Barr ${ }^{7}$
}

${ }^{1} \mathrm{MRC/CSO}$ Social and Public Health Sciences Unit, University of Glasgow, Glasgow, UK ${ }^{2}$ Department of Disease Control, London School of Hygiene and Tropical Medicine, London, UK ${ }^{3}$ Institute of Infection, Veterinary and Ecological Sciences,

University of Liverpool, Liverpool UK

${ }^{4}$ Institute of Health \& Wellbeing, University of Glasgow, Glasgow, UK

${ }^{5}$ Primary Care and Public Health Imperial College London, London, UK

${ }^{6}$ Department of Public Health, University of Copenhagen Faculty of Health and Medical Sciences, Copenhagen K, Denmark

${ }^{7}$ Public Health and Policy, University of Liverpool, Liverpool, UK

\section{Correspondence to}

Professor Srinivasa Vittal Katikireddi, University of Glasgow MRC/CSO Social and Public Health Sciences Unit, Glasgow G3 7HR, UK; vittal.katikireddi@glasgow.ac.uk

Received 16 November 2020 Revised 20 February 2021 Accepted 8 April 2021 Published Online First 21 April 2021

\section{ABSTRACT}

Minority ethnic groups have been disproportionately affected by the COVID-19 pandemic. While the exact reasons for this remain unclear, they are likely due to a complex interplay of factors rather than a single cause. Reducing these inequalities requires a greater understanding of the causes. Research to date, however, has been hampered by a lack of theoretical understanding of the meaning of 'ethnicity' (or race) and the potential pathways leading to inequalities. In particular, quantitative analyses have often adjusted away the pathways through which inequalities actually arise (ie, mediators for the effect of interest), leading to the effects of social processes, and particularly structural racism, becoming hidden. In this paper, we describe a framework for understanding the pathways that have generated ethnic (and racial) inequalities in COVID-19. We suggest that differences in health outcomes due to the pandemic could arise through six pathways: (1) differential exposure to the virus; (2) differential vulnerability to infection/disease; (3) differential health consequences of the disease; (4) differential social consequences of the disease; (5) differential effectiveness of pandemic control measures and (6) differential adverse consequences of control measures. Current research provides only a partial understanding of some of these pathways. Future research and action will require a clearer understanding of the multiple dimensions of ethnicity and an appreciation of the complex interplay of social and biological pathways through which ethnic inequalities arise. Our framework highlights the gaps in the current evidence and pathways that need further investigation in research that aims to address these inequalities.

\section{INTRODUCTION}

Minority ethnic groups have been disproportionately affected by the COVID-19 pandemic, with the clearest evidence from the UK and the USA. ${ }^{1-4}$ While the exact reasons for this remain unclear, they are likely due to a complex interplay of a number of factors rather than a single cause. Reducing these inequalities requires a greater understanding of the causes. Research to date, however, has been hampered by a lack of theoretical understanding of the meaning of 'ethnicity' or the potential pathways leading to ethnic inequalities.
In this paper, we describe a framework for understanding the pathways that have generated ethnic inequalities in COVID-19-to our knowledge, the first of its kind. Current research provides only a partial understanding of some of these pathways. Future research and action will require a clearer understanding of the complex dimensions of ethnicity and an appreciation of the complex interplay of social and biological pathways through which ethnic inequalities arise. Our framework highlights the gaps in the current evidence and pathways that need further investigation in research that aims to address these inequalities.

\section{UNDERSTANDING ETHNICITY}

Ethnicity is socially constructed. ${ }^{5}$ It can be defined as a 'social group a person belongs to, and either identifies with or is identified with by others, as a result of a mix of cultural and other factors including language, diet, religion, ancestry, and physical features traditionally associated with race'. ${ }^{6}$ Ethnicity is therefore a complex concept which includes multiple dimensions including country of birth, language, religion and culture. Although it is socially constructed, it may be associated with biological attributes such as skin colour, that influence the unequal treatment of people within racist societies. The act of categorising people into ethnic groups is a social process, influenced by particular social, cultural and historical contexts. For this reason, ethnic categories differ across the world, with the same term often referring to different groups of people-for example, the term 'Asian' is often understood as referring mainly to East Asian people in the USA whereas in the UK the same term is typically interpreted as including people from the Indian subcontinent. ${ }^{5}$

In this paper, we therefore use the term ethnicity throughout but include the concept of race within this term (as defined above) and consider racial inequalities as core to ethnic inequalities. This reflects a tradition in the UK of focusing on ethnic inequalities in health, but we acknowledge inter-related dimensions on inequality are often given greater emphasis in different countries. For example, in the USA the term race is more widely used, with the socially constructed nature of racial categories also explicitly acknowledged by public health researchers. ${ }^{7}$ Similarly, in many European countries outside of the UK the health of migrants 
(classified on the basis of country of birth) has often been the focus of research, rather than minority ethnic groups-at least in part due to a lack of data collection on ethnicity. While we use terminology related to ethnicity throughout the remainder of the paper, we believe our framework and the arguments expressed broadly apply to inequalities related to migrant status and similar related inequalities. The use of ethnicity also allows us to include inequalities experienced within broader racial groups-for example, by white traveller and gypsy communities across Europe. ${ }^{8}$ We also note that ethnic groups that experience disadvantage can be numerical majorities in some countries and our use of the term minority ethnic also refers to relative power within society. ${ }^{9}$

While not all minority ethnic groups in all countries experience worse health than the majority ethnic group, ${ }^{10}$ differences in health across ethnic groups, in terms of both morbidity and mortality, have been repeatedly documented in the UK and other countries. ${ }^{11}$ It is important to note that the health and related experiences of minority ethnic groups are not homogenous, with different patterns seen depending on which health outcomes are studied. ${ }^{5}$ While understanding by current researchers has largely moved on from racist scientific thinking of the 19th century that narrowly viewed these differences through a biological lens, ${ }^{7}$ this is not universally the case. ${ }^{12}$ The multiple dimensions of ethnicity influence health through their interaction with wider social processes. In the past, social disadvantage, and particularly experiences of racism, have been downplayed as explanations for these differences. However, there is now increasing recognition of the role of structural racism. Processes of racialisation are contingent on socio-historical context, such that some groups may be more or less racialised at different times. For example, white Irish people living in the UK were subject to substantial and overt racism in the early 20th century, with other racial groups (such as white Eastern European and travelling community groups) being more targeted at the end of the century. ${ }^{13}$

Structural racism has been defined as 'the macrolevel systems, social forces, institutions, ideologies, and processes that interact with one another to generate and reinforce inequities among racial and ethnic groups'. ${ }^{14}$ The term draws attention to the way these ethnic inequalities arise not only from the intended actions of individuals, but rather from broader societal mechanisms. For example, historical experiences of minority ethnic groups and long-term discrimination may lead to a higher proportion working in lower paid jobs on insecure contracts without sickness benefits and in public-facing occupations, living in crowded housing conditions, and having fewer resources for health (eg, education, income). ${ }^{15}$ These factors are likely to increase psychosocial stress, mental health problems and harmful health behaviours (eg, smoking, poor diet and physical inactivity). There is also a wealth of evidence documenting inequalities faced by minority ethnic groups in accessing quality healthcare. ${ }^{5}$ Healthcare planning may not take into account different experiences, perceptions and expectations of ethnic minorities, and therefore health services may not meet the needs of some ethnic groups-further widening inequalities. ${ }^{5}$ Reported experiences of racial discrimination are also associated with poorer health. ${ }^{16}$ This includes both interpersonal racism (which refers to discriminatory actions between individuals) and institutional racism (when discriminatory policies and practices are embedded in organisations). While ethnic inequalities in health are often linked to socioeconomic differences, they are not entirely explained by these factors due to the experiences of discrimination and intersecting inequalities within and across social groups.
Studies of ethnic differences in health have not paid sufficient attention to the social processes that give rise to these inequalities. Often studies include 'ethnic group' as one of many variables in regression models 'controlling or adjusting' for clinical, social and economic factors that are in fact important explanations of ethnic inequalities. This process has resulted in researchers sometimes erroneously concluding that ethnic inequalities do not exist (eg, see Yehia and colleagues ${ }^{17}$ ). Adjusting away the pathways through which inequalities actually arise (ie, mediators) can lead to the effects of social processes, and particularly structural racism, becoming hidden. This 'black box epidemiology', which has been often used in recent studies of ethnic inequalities in COVID $-19{ }^{18}$ has been rightly criticised for ignoring the theory underpinning analyses. ${ }^{19}$ More theory-informed analyses can help yield more informative insights.

\section{FRAMEWORK TO UNDERSTAND ETHNIC INEQUALITIES IN THE HEALTH IMPACTS OF THE PANDEMIC}

To inform analyses of ethnic inequalities in the health impacts of the COVID-19 crisis, we present a framework of the potential mechanisms and pathways that could contribute to health differences between ethnic groups (figure 1). We build on a wellestablished framework for studying health inequalities ${ }^{2021}$ which distinguished the individual proximate causes of disease from their societal causes and highlighted the potential importance of differential exposure to causes of disease, differential vulnerability to their effects and differential consequences of disease. In our model, we suggest that differences in health outcomes due to COVID-19 could occur at multiple stages: from exposure to the virus, development of disease, and through the indirect impacts of control measures and management of an individual with COVID-19. At each step, ethnic inequalities could develop through social and economic mechanisms which have biological effects. A comprehensive understanding of these pathways will help identify targets for policy interventions, as well as future research. We provide a brief introduction to each element of the framework, drawing on relevant studies to illustrate how it might be relevant to ethnic inequalities in health arising from the pandemic. We note that we have not conducted a systematic assessment of the evidence base in relation to each of these pathways and we therefore provide these studies for illustrative purposes only.

\section{Differential exposure}

Minority ethnic groups could experience greater exposure to the virus and therefore higher risk of infection, which could relate to the frequency of contact or the potential infective dose of each contact. For example, working in specific occupations (eg, health and social care workers, transport workers) or living in overcrowded housing could lead to being in contact with potentially infected persons more frequently and for a longer duration potentially leading to a higher viral load. ${ }^{22} 23$ Recent findings from a representative English infection survey found evidence that all minority ethnic groups studied were more likely to have serological evidence of previous SARS-CoV-2 infection compared with the majority white British population. ${ }^{24}$

\section{Differential vulnerability to infection/disease}

Minority ethnic groups could be more likely to develop disease once exposed. This could result from differences in nutritional status, comorbidities and immune response, which themselves could be driven by stress or environmental conditions, such as air pollution. There is considerable evidence that incidence of 


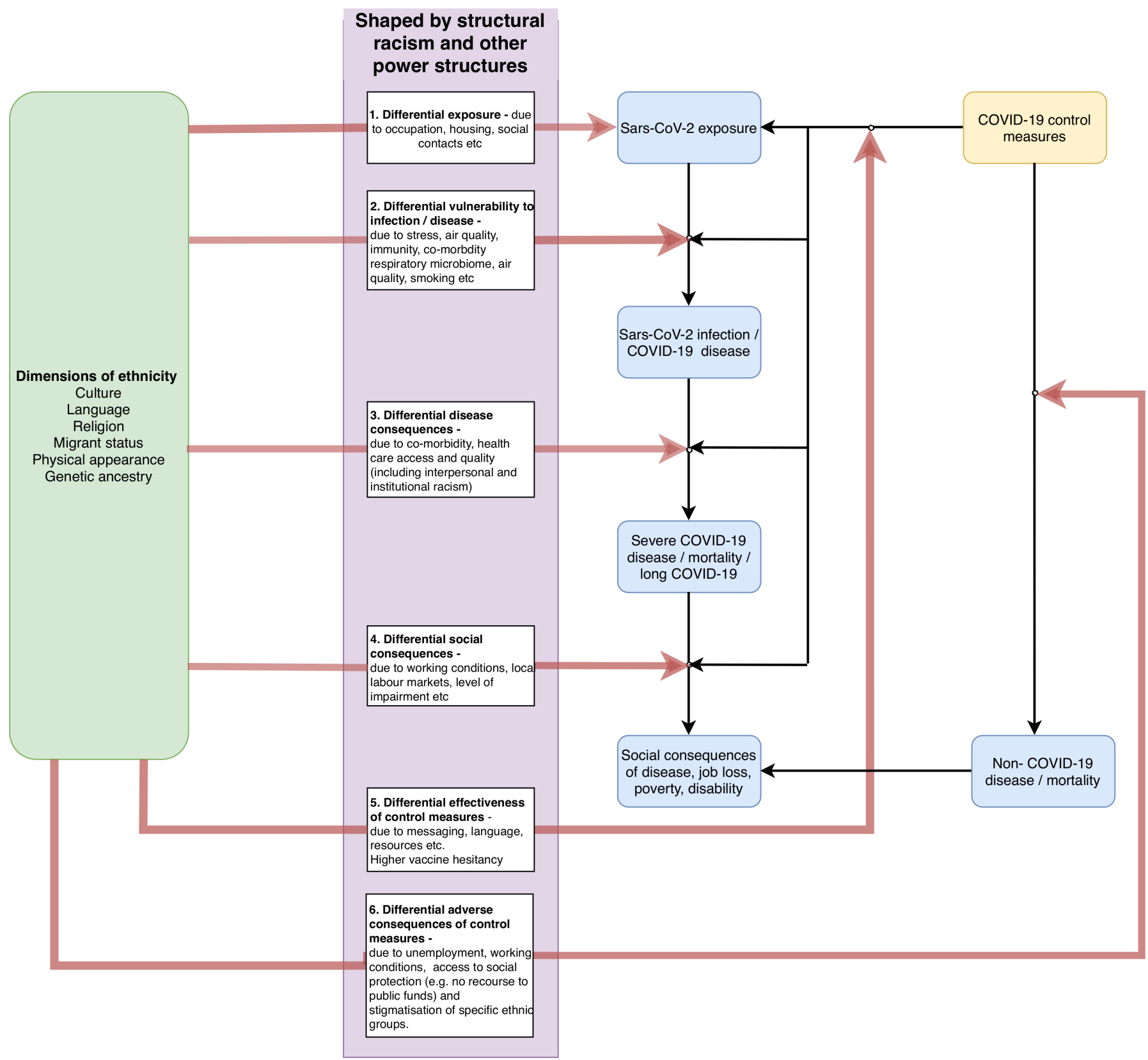

Figure 1 A framework for understanding pathways underpinning ethnic inequalities in COVID-19 and potential targets for policy.

clinically important disease (reflected by positive symptomatic tests or hospitalisations) are higher in many minority ethnic groups. ${ }^{218}$ However, it is not clear if this merely reflects greater exposure or differences in vulnerability to developing disease. To differentiate vulnerability to the disease from greater exposure, analysis would need to compare ethnic differences in the risk of reporting symptomatic disease among both symptomatic and asymptomatically infected people.

\section{Differential disease consequences}

Of those with disease, some minority ethnic groups may be more likely to develop severe disease, require mechanical ventilation, experience complications and potentially die. This could, for example, be due to differences in underlying comorbidities or differential access to healthcare between ethnic groups. ${ }^{23} \mathrm{~A}$ large cohort study of nearly 35000 UK hospitalisations found a $30 \%$ increased relative risk of critical care admission and mechanical ventilation among people from South Asian, black or minority ethnic groups and this relationship was still present after adjusting for age and sex. ${ }^{25}$ After accounting for some potential explanations of this increased risk (such as comorbidities like diabetes), minority ethnic groups were still more likely to require critical care and mechanical ventilation than white groups. Different ethnic groups may be at risk of longer term health consequences, such as greater risks of 'long COVID-19' (also referred to as post-COVID-19 syndrome), ${ }^{26}$ but evidence is currently limited.

\section{Differential social consequences}

Minority ethnic groups may also experience differential social consequences following recovery from COVID-19 disease. COVID-19 disease may lead to long-lasting disability that results in job loss and future loss of earnings due to poor health. One important reason for a potential disproportionate impact 
on minority ethnic groups is the often higher levels of insecure employment (such as self-employment or being on 'zero hours' contracts) that were already experienced prepandemic. Contracting COVID-19 disease, and especially 'long COVID19 ', could lead to minority ethnic people being more likely to experience job loss and poverty. However, empirical data remain relatively scarce on the social consequences of the disease ${ }^{26}$ and further research is required to address this gap.

\section{Differential effectiveness of control measures}

Public health interventions designed to control the pandemic may also, in themselves generate ethnic health inequalities, as their impact on risk of exposure, vulnerability and consequences (pathways 1-4) may be different for some ethnic groups, sometimes also referred to as intervention-generated inequalities. ${ }^{27}$ We believe conceptualising of the differential effectiveness of control measures as a distinct pathway is important given the necessity of understanding the impacts of public health actions. These unintended intervention-generated inequalities may operate through changing the risk across the other four pathways. For example, health communications which have not been culturally adapted to their target audiences may be less effective in some groups. ${ }^{28}$ Control measures that are inequitably implemented could mean that people in the same occupation experience different exposure risks. There is evidence to suggest that minority ethnic healthcare workers were less likely to be able to access personal protective equipment. ${ }^{29}$ In contrast, lockdown measures appeared to reduce COVID-19 mortality more among some minority ethnic groups than the majority population. ${ }^{30}$ The availability of effective vaccines is hugely welcome but could further exacerbate ethnic inequalities. For example, some minority ethnic groups (such as black and Pakistani/Bangladeshi ethnic groups) have higher levels of vaccine hesitancy in the UK. ${ }^{31}$ Importantly, differences in vaccine hesitancy reflect broader societal processes, such as the dominant communication strategies used in vaccination programmes and understandable concerns arising from longstanding experiences of discrimination (such as the Windrush scandal which led to the illegal deportation of black British citizens).

\section{Differential adverse consequences of control measures}

Social and economic impacts of pandemic control measures (such as loss of income) may also disproportionately affect disadvantaged groups more and these impacts may affect non-COVID-19 health outcomes. ${ }^{32}$ Evidence already suggests that some minority ethnic groups have disproportionately experienced unemployment during the initial lockdown period and a greater increase in psychological distress, exacerbating existing ethnic inequalities in mental health. ${ }^{33} 34$

All of these six pathways arise from the wider social and political context that drive ethnic and other social inequalities, including structural racism and other power imbalances across society. Furthermore, multiple risks can affect multiple pathways-for example, poor working conditions might affect both the potential for differential exposure and experiencing differential consequences of control measures. Despite this, an understanding of the pathways driving ethnic inequalities can help identify policy targets.

\section{CONCLUSIONS}

The COVID-19 pandemic has highlighted existing health inequalities among ethnic minority groups and exacerbated them. This has led to an increase in research studies to understand ethnic inequalities in health, but many research studies are based on a constrained and limited understanding of ethnicity and the potential pathways generating differences in health between ethnic groups. Ethnicity is a complex, multidimensional social construct and health differences between some ethnic groups largely reflect social pathways, embedded within the unequal power relationships that propagate inequalities. It is inappropriate for researchers to investigate ethnic groupings like biomarker or biomedical variables in naïve multivariable analysis that is not theoretically informed. In particular, overadjustment for mediating variables can lead to misleading interpretations, provide little insight to inform policy and practice and may ultimately have harmful real-world consequences. ${ }^{35}$ The unequal impacts of the pandemic can be mitigated, through more comprehensive and evidence informed action at each of the pathways we outlined above. This requires research that elucidates how specific dimensions of ethnicity differentially affect the mechanisms of differential exposure, vulnerability and consequences, identifying the most effective policy entry points to reduce ethnic inequalities in health. Our framework is a first step towards encouraging clearer thinking on ethnic inequalities in COVID-19 and we welcome feedback, anticipating that refinements will be needed over time.

\section{What is already known on this topic}

Minority ethnic populations have experienced disproportionate harms during the COVID-19 pandemic.

- Considerable research is ongoing to understand the reasons for the greater risks being experienced, but a lack of theoretical underpinning for epidemiological analyses is often leading researchers to make erroneous conclusions.

\section{What this study adds}

- We present a framework for understanding the drivers of ethnic inequalities in COVID-19 harms, highlighting the multitude of mechanisms through which structural racism and power imbalances operate.

- Applying a theoretical framework can help policymakers and researchers develop more valid conclusions and ultimately better inform public health policies to mitigate adverse consequences of the pandemic on ethnic inequalities in health.

Twitter Srinivasa Vittal Katikireddi @vkatikireddi and Ben Barr @benj_barr

Contributors SVK and BB conceived the idea for the paper. SVK wrote the first draft. All authors contributed to the ideas expressed, critically revised the manuscript and agreed to the final version.

Funding SVK and RD acknowledge funding from the Medical Research Council (MC_UU_00022/2). SVK additionally acknowledges funding from the Scottish Government Chief Scientist Office (SPHSU17) and an NRS Senior Clinical Fellowship (SCAF/15/02). SL is supported by grants from the Wellcome Trust. EDC acknowledges funding from the NIHR i4i Programme (II-LA-0216-20002), HTA Programme (15/188/42, 17/136/13), EME Programme (NIHR129960) and H2020 (Project No. 848196). CLN is funded by a Medical Research Council Fellowship (MR/R024774/1). KK is supported by the National Institute for Health Research (NIHR) Applied Research Collaboration East Midlands (ARC EM) and the NIHR Leicester Biomedical Research Centre (BRC). BB, is supported by National Institute for Health Research (NIHR) Applied Research Collaboration North West Coast (ARC NWC, NIHR200182) and NIHR School for Public Health Research (PD-SPH-2015-10062). The funders had no involvement in the writing or decision to submit the manuscript. The views expressed in this publication are those of the author(s) and not necessarily those of the NIHR or the Department of Health and Social Care. 
Competing interests SVK, SL, EDC, KK and BB are members of the UK Government's Scientific Advisory Group on Emergencies (SAGE) subgroup on ethnicity and COVID-19. SVK is Co-chair of the Scottish Government's Expert Reference Group on ethnicity and COVID-19. SL is currently seconded to the UK's Government Office of Science. KK is Director for the University of Leicester Centre for BME Health, Trustee of the South Asian Health Foundation, national NIHR ARC lead for Ethnicity and Diversity and a member of Independent SAGE. All authors write in an independent capacity and the views expressed do not necessarily represent any government or funding organisation.

Patient consent for publication Not required.

Provenance and peer review Not commissioned; externally peer reviewed.

Data availability statement Data sharing not applicable as no datasets generated and/or analysed for this study.

Open access This is an open access article distributed in accordance with the Creative Commons Attribution 4.0 Unported (CC BY 4.0) license, which permits others to copy, redistribute, remix, transform and build upon this work for any purpose, provided the original work is properly cited, a link to the licence is given, and indication of whether changes were made. See: https://creativecommons.org/ licenses/by/4.0/

\section{ORCID iDs}

Srinivasa Vittal Katikireddi http://orcid.org/0000-0001-6593-9092

Sham Lal http://orcid.org/0000-0002-3650-1398

Claire L Niedzwiedz http://orcid.org/0000-0001-6133-4168

Ruth Dundas http://orcid.org/0000-0002-3836-4286

Finn Diderichsen http://orcid.org/0000-0002-9998-4972

\section{REFERENCES}

1 England PH. COVID-19: review of disparities in risks and outcomes. London: Public Health England, 2020.

2 Niedzwiedz CL, O'Donnell CA, Jani BD, et al. Ethnic and socioeconomic differences in SARS-CoV-2 infection: prospective cohort study using UK Biobank. BMC Med 2020;18:160

3 Raharja A, Tamara A, Kok LT. Association between ethnicity and severe COVID-19 disease: a systematic review and meta-analysis. J Racial Ethn Health Disparities 2020. doi:10.1007/s40615-020-00921-5. [Epub ahead of print: 12 Nov 2020].

4 Sze S, Pan D, Nevill CR, et al. Ethnicity and clinical outcomes in COVID-19: a systematic review and meta-analysis. EClinicalMedicine 2020;29:100630.

5 Bhopal RS. Migration, ethnicity, race, and health in multicultural societies. Oxford University Press: Oxford, 2014

6 Bhopal R. Glossary of terms relating to ethnicity and race: for reflection and debate. $J$ Epidemiol Community Health 2004;58:441-5.

7 Krieger N. Refiguring "race": epidemiology, racialized biology, and biological expressions of race relations. Int I Health Serv 2000;30:211-6.

8 McFadden A, Siebelt L, Gavine A, et al. Gypsy, Roma and traveller access to and engagement with health services: a systematic review. Eur J Public Health 2018:28:74-81.

9 Johnson MRD, Bhopal RS, Ingleby JD, et al. A glossary for the first world Congress on migration, ethnicity, race and health. Public Health 2019;172:85-8.

10 Bhopal RS, Gruer L, Cezard G, et al. Mortality, ethnicity, and country of birth on a national scale, 2001-2013: a retrospective cohort (Scottish health and ethnicity linkage study). PLoS Med 2018;15:e1002515.

11 Nazroo JY. The structuring of ethnic inequalities in health: economic position, racial discrimination, and racism. Am J Public Health 2003:93:277-84.

12 Yudell M, Roberts D, DeSalle R, et al. Taking race out of human genetics. Science 2016:351:564-5.

13 Rzepnikowska A. Racism and xenophobia experienced by Polish migrants in the UK before and after Brexit vote. J Ethn Migr Stud 2019;45:61-77.
14 Gee GC, Ford CL. Structural racism and health inequities: old issues, new directions. Du Bois Rev 2011:8:115.

15 Karlsen S, Nazroo JY, Smith NR. Ethnic, religious and gender differences in Intragenerational economic mobility in England and Wales. Sociology 2020;54:883-903.

16 Priest N, Paradies Y, Trenerry B, et al. A systematic review of studies examining the relationship between reported racism and health and wellbeing for children and young people. Soc Sci Med 2013;95:115-27.

17 Yehia BR, Winegar A, Fogel R, et al. Association of race with mortality among patients hospitalized with coronavirus disease 2019 (COVID-19) at 92 US hospitals. JAMA Netw Open 2020;3:e2018039-e:e2018039.

18 Williamson EJ, Walker AJ, Bhaskaran K, et al. Factors associated with COVID-19related death using OpenSAFELY. Nature 2020;584:430-6.

19 Tennant PWG, Murray EJ. The quest for timely insights into COVID-19 should not come at the cost of scientific rigor. Epidemiology 2021;32:e2.

20 Diderichsen F, Andersen I, Manuel C, et al. Health inequality--determinants and policies. Scand J Public Health 2012:40:12-105.

21 Diderichsen F, Augusto LGdaS, Perez B. Understanding social inequalities in Zika infection and its consequences: a model of pathways and policy entry-points. Glob Public Health 2019;14:675-83.

22 Mutambudzi M, Niedwiedz C, Macdonald EB, et al. Occupation and risk of severe COVID-19: prospective cohort study of 120075 UK Biobank participants. Occup Environ Med 2021;78:307-14

23 Daras K, Alexiou A, Rose TC, et al. How does vulnerability to COVID-19 vary between communities in England? developing a small area vulnerability index (SAVI). J Epidemiol Community Health 2021;75:729-34.

24 Office for National Statistics. Coronavirus (COVID-19) infection survey: characteristics of people testing positive for COVID-19 in England, August 2020. London, 2020.

25 Harrison EM, Docherty AB, Barr B. Ethnicity and outcomes from COVID-19: the ISARIC CCP-UK prospective observational cohort study of hospitalised patients. SSRN preprint 2020.

26 Yelin D, Wirtheim E, Vetter P, et al. Long-Term consequences of COVID-19: research needs. Lancet Infect Dis 2020:20:1115-7.

27 Lorenc T, Petticrew M, Welch V, et al. What types of interventions generate inequalities? Evidence from systematic reviews. J Epidemiol Community Health 2013:67:190-3.

28 Davidson EM, Liu JJ, Bhopal R, et al. Behavior change interventions to improve the health of racial and ethnic minority populations: a tool kit of adaptation approaches. Milbank Q 2013;91:811-51

29 Nguyen LH, Drew DA, Graham MS, et al. Risk of COVID-19 among front-line healthcare workers and the general community: a prospective cohort study. Lancet Public Health 2020:5:e475-83.

30 Ayoubkhani D, Nafilyan V, White C. Ethnic minority groups in England and Wales factors affecting the size and timing of elevated COVID-19 mortality: a retrospective cohort study linking Census and death records. medRxiv 2020.

31 Robertson E, Reeve KS, Niedzwiedz CL, et al. Predictors of COVID-19 vaccine hesitancy in the UK household longitudinal study. Brain Behav Immun 2021. doi:10.1016/j.bbi.2021.03.008. [Epub ahead of print: 11 Mar 2021].

32 Douglas M, Katikireddi SV, Taulbut M, et al. Mitigating the wider health effects of covid-19 pandemic response. BMJ 2020;369:m1557.

$33 \mathrm{Hu}$ Y. Intersecting ethnic and native-migrant inequalities in the economic impact of the COVID-19 pandemic in the UK. Res Soc Stratif Mobil 2020;68:100528.

34 Niedzwiedz CL, Green MJ, Benzeval M, et al. Mental health and health behaviours before and during the initial phase of the COVID-19 lockdown: longitudinal analyses of the UK household longitudinal study. J Epidemiol Community Health 2021;75:224231

35 Katikireddi SV, Valles SA. Coupled Ethical-Epistemic analysis of public health research and practice: categorizing variables to improve population health and equity. Am J Public Health 2015;105:e36-42. 